\title{
THE FOURIER TRANSFORM OF AN UNBOUNDED SPECTRAL DISTRIBUTION
}

\author{
BRIAN KRITT
}

\begin{abstract}
The Fourier transform of an unbounded spectral distribution is studied: An explicit integral representation is obtained; connections are drawn to the associated generalized scalar operator. It is proved that every generalized pseudo-hermitian operator is the infinitesimal generator of a temperate $C_{0}$ group.
\end{abstract}

Introduction. In [6] the author introduced a theory of unbounded spectral distributions in Banach spaces, and a corresponding theory of the generalized scalar operators which they represent. Properties of these objects were studied, culminating in a spectral mapping theorem [6, Theorem 4]. In this paper we study the Fourier transform of an unbounded spectral distribution, deriving an explicit integral representation, as well as growth estimates at infinity. The case of real support is considered in some detail, leading to the proof that every generalized pseudo-hermitian operator (i.e., generalized scalar with real spectrum) is the infinitesimal generator of a temperate group. This result generalizes the corresponding result for bounded operators proved in [5].

In this paper, all definitions are as in [6].

\section{Integral representation of the Fourier transform.}

THEOREM 1 (EXTENSION OF SPECTRAL DISTRIBUTIONS). Let $T$ be $a$ spectral distribution [6, Definition 2] in a Banach space $X$. Then

(a) for each function from the space $\mathscr{B}\left(R^{2}\right)$ of $C^{\infty}$ functions with bounded derivatives, and for each $x \in X$, limit ${ }_{n} T_{f \varphi_{n}} x$ exists, where $\left\{\varphi_{n}\right\}$ is a sequence of test functions as in [6, Definition 2(c)].

(b) If we define $T_{f} x \equiv \operatorname{limit}_{n} T_{f \varphi_{n}} x$ for all $x \in X$, then $T_{f}$ is in the space $\mathscr{L}(X)$ of all bounded linear operators on $X$, and the correspondence $f \rightarrow T_{f}$ is a continuous linear mapping of $\mathscr{B}\left(R^{2}\right)$ into $\mathscr{L}(X)$.

(c) $T_{f g}=T_{f} T_{y}$ for all $f, g \in . \mathscr{B}\left(R^{2}\right)$.

(d) The operator $T_{f}$ is independent of the sequence $\left\{\varphi_{n}\right\}$

Proof. (a) Define a double sequence of functions $\psi_{n m} \equiv f\left(\varphi_{n}-\varphi_{m}\right)$. Then $\left\{\psi_{n m}\right\}$ form a $\mathscr{B}\left(R^{2}\right)$-bounded subset of $\mathscr{L}\left(R^{2}\right)$ (cf. [3, p. 91] for the

Received by the editors October 20, 1971.

AMS 1970 subject cle ssifications. Primary 47A65, 47A60, 47B40; Secondary 46F99.

(.) American Mathematucal Society 1972 
topology of $\mathscr{B}\left(R^{2}\right)$ ). And $\inf \left\{|\lambda|: \lambda \in\right.$ support $\left.\psi_{n m}\right\} \rightarrow \infty$ as $n, m \rightarrow \infty$. Since $T$ strongly vanishes to power 0 at infinity (cf. [6, Definition 1]), $T_{f \varphi_{n}} x-T_{f \varphi_{m}} x=T_{\varphi_{n m}} x \rightarrow 0$.

(b) $T_{f}$ is everywhere defined and linear on $X$, by (a). Since $\left\|T_{f \varphi_{n}}\right\|$ are bounded, $T_{f}$ is bounded. It is clear that the correspondence $f \rightarrow T_{f}$ is linear. Let $\mathscr{F}$ be a bounded subset of $\mathscr{B}\left(R^{2}\right)$. Then the set $\left\{\varphi_{n} f: f \in \mathscr{F}\right\}$ is a $\mathscr{B}\left(R^{2}\right)$-bounded subset of $\mathscr{D}\left(R^{2}\right)$. Thus $\left\|T_{f \varphi_{n}}\right\|$ are bounded; i e., there is an $M>0$ such that $\left\|T_{f \varphi_{n}} x\right\| \leqq M\|x\|$ for all $x \in X, f \in \mathscr{F}$, and $n$. Letting $n \rightarrow \infty$, we get $\left\|T_{f}\right\| \leqq M$ for all $f \in \mathscr{F}$. Since $\mathscr{B}\left(R^{2}\right)$ is bornological [3, p. 222], the correspondence $f \rightarrow T_{f}$ is continuous.

(c) $T_{f g \varphi_{n}} x=\operatorname{limit}_{m} T_{f g \varphi_{n}} T_{\varphi_{m}} x=\operatorname{limit}_{m} T_{f \varphi_{n} g \varphi_{m}} x=\operatorname{limit}_{m} T_{f \varphi_{n}} T_{g \varphi_{m}} x=$ $T_{f i \varphi_{n}} T_{g} x$. Taking the limit as $n \rightarrow \infty$, we get $T_{f g} x=T_{f} T_{g} x$ for all $x \in X$.

(d) If $\left\{\psi_{n}\right\}$ is another such sequence, then $T_{f \varphi_{n}} x-T_{f \psi_{n}} \gamma=T_{f\left(\varphi_{n}-\psi_{n}\right)} x$. Since $\left\{f\left(\varphi_{n}-\psi_{n}\right)\right\}$ is a $\mathscr{B}\left(R^{2}\right)$-bounded subset of $\mathscr{D}\left(R^{2}\right)$ for which the supports tend to infinity, the conclusion follows from the fact that $T$ strongly vanishes to power 0 at infinity.

REMARKS. For the function $f \in \mathscr{B}\left(R^{2}\right)$ which is identically $1, T_{f}=I$, the identity operator in $X$, by the normalization condition on $T[6$, Definition 2(c)].

The extension of $T$ to $\mathscr{B}_{0}\left(R^{2}\right)$ (functions in $\mathscr{B}\left(R^{2}\right)$ vanishing at infinity together with all derivatives) is unique as a continuous extension of $T$; and to $\mathscr{B}\left(R^{2}\right)$, unique as a multiplicative extension.

Since $T$ is continuous with respect to the $\mathscr{B}\left(R^{2}\right)$ topology, it is tempered. Thus $T$ has a Fourier transform $\hat{T}$ defined by the formula $\hat{T}_{\varphi} \equiv T_{\hat{\varphi}}$ for all $\varphi \in \mathscr{S}\left(R^{2}\right) \subset \not \mathscr{B}\left(R^{2}\right)$.

Theorem 2. Let $T$ be a spectral distribution in $X$. For $(\xi, \eta) \in R^{2}$, define

$$
f_{\xi, \eta}(\lambda) \equiv \exp (-i(\operatorname{Re} \lambda \cdot \xi+\operatorname{Im} \lambda \cdot \eta)) \quad \text { for all } \lambda \Leftrightarrow R^{2} \equiv C \text {. }
$$

Then

(a) $f_{\xi, \eta}(\cdot) \in \mathscr{B}\left(R^{2}\right)$;

(b) the function $F$ from $R^{2}$ into $\mathscr{L}^{\prime}(X)$ defined by $F(\xi, \eta) \equiv T_{f_{\xi, \eta}}$ is strongly continuous in $(\xi, \eta) \in R^{2}$;

(c) $F(0,0)=I$;

(d) $\hat{T}_{\varphi} x=\int_{R^{2}} \varphi(\xi, \eta) F(\xi, \eta) x d \xi d \eta$ for all $\varphi \in \mathcal{C}\left(R^{2}\right) . x \in X$;

(e) there is a $C>0$ and a positive integer $N$ such that

$$
\|F(\xi, \eta)\| \leqq C(1+|\xi+i \eta|)^{v} \text { for all }(\xi, \eta) \in R^{2} .
$$

Proof. (a) and (c) are clear.

(b) Since $F(\xi, \eta) x=\lim _{n} T_{\varphi_{n} f_{\xi}, \eta} x, F$ is strongly Bochner measurable; in fact, the functions $F_{n}(\xi, \eta) \equiv T_{\varphi_{n} f \xi, \eta}$ are even analytic. Let $G(\xi) \equiv$ $F(\xi, 0)$. Then $G\left(\xi_{1}+\xi_{2}\right)=G\left(\xi_{1}\right) G\left(\xi_{2}\right)$, and $G$ is strongly measurable, so 
Lemma 3 of $[1$, p. 616] implies $G$ is strongly continuous. Similarly, $H(\eta) \equiv$ $F(0, \eta)$ is strongly continuous. Now $F(\xi, \eta)=G(\xi) H(\eta)=H(\eta) G(\xi)$; let $x \in X, \xi_{n} \rightarrow \xi, \eta_{n} \rightarrow \eta$. Then $H\left(\eta_{n}\right) x$ are bounded, so the Banach-Steinhaus Theorem implies $\left\|H\left(\eta_{n}\right)\right\|$ are bounded by some number $M$. Thus

$$
\begin{aligned}
\| F\left(\xi_{n},\right. & \left.\eta_{n}\right) x-F(\xi, \eta) x \| \\
& \leqq\left\|H\left(\eta_{n}\right)\left[G\left(\xi_{n}\right)-G(\xi)\right] x\right\|+\left\|\left[H\left(\eta_{n}\right)-H(\eta)\right] G(\xi) x\right\| \\
& \leqq M\left\|\left[G\left(\xi_{n}\right)-G(\xi)\right] x\right\|+\left\|\left[H\left(\eta_{n}\right)-H(\eta)\right] G(\xi) x\right\|,
\end{aligned}
$$

which implies the strong continuity of $F$.

(d) Fix $x \in X, x^{*} \in X^{*}$ (dual of $X$ ). Let $\left\{u_{n}\right\}$ be the distributions defined by $u_{n}(\varphi)=x^{*} T_{\varphi_{n} \varphi} x$ for all $\varphi \in \mathscr{D}\left(R^{2}\right)$, where $\varphi_{n}$ are as usual. Then $u_{n}$ has compact support. and [2. Theorem 1.7.5] implies

$$
\begin{aligned}
\hat{u}_{r}(\varphi) & =\int_{R^{2}} \varphi(\xi, \eta) u_{n}\left(f_{\xi, \eta}\right) d \xi d \eta \\
& =\int_{R^{2}} \varphi(\xi, \eta)\left[x^{*} F_{n}(\xi, \eta) x\right] d \xi d \eta \\
& \quad \cdot \int_{R^{2}} q(\xi, \eta) x^{*} F(\xi, \eta) x d \xi d \eta
\end{aligned}
$$

by the Dominated Convergence Theorem, since $\left\|F_{n}(\xi, \eta) x\right\| \leqq C\|F(\xi, \eta) x\|$ (where $\left.C=\sup _{n} \| \tau_{\varphi_{n}} i\right)$. Of course $\|F(\xi, \eta) x\|$ is bounded on the support of $\varphi$ by (b). On the other hand $\hat{u}_{n}(\varphi)=u_{n}(\hat{\varphi})=x^{*} T_{\varphi_{n} \hat{\varphi}} x \rightarrow{ }_{n} x * T_{\hat{\varphi}} x$. Thus

$$
x^{*} T_{\hat{\varphi}} x=\int_{R^{2}} \psi(\xi, \eta) x^{*} F(\xi, \eta) x d \xi d \eta=x^{*} \int_{R^{2}} \varphi(\xi, \eta) F(\xi, \eta) x d \xi d \eta
$$

from which (d) is an immediate consequence.

(e) By the continuity of $T$ on $\mathscr{B}\left(R^{2}\right)$ there is a constant $C$ and a positive integer $p$ such that

$$
\| T_{\varphi p}: \leqq C \cdot \sum_{|x| \leqq p}\left|D^{\alpha} \varphi\right|_{\infty} \quad \text { for all } q^{\prime} \in \mathscr{B}\left(R^{2}\right),
$$

where $\alpha \equiv\left(\alpha_{1}, \alpha_{2}\right)$ is a bi-index, $|\alpha| \equiv \alpha_{1}+\alpha_{2}$, and $D^{\alpha} \equiv \partial^{|\alpha|} /\left(\partial x_{1}\right)^{\alpha_{1}}\left(\partial x_{2}\right)^{\alpha_{2}}$. (Here $x_{1} \equiv \operatorname{Re} \lambda, x_{i 2} \equiv \operatorname{Im} \lambda$.)

Now

$$
\begin{aligned}
\|F(\xi, 0)\| & =\left\|T_{f_{\xi, 0}}\right\| \leqq C \sum_{|\alpha| \leqq p}\left|D^{\alpha} f_{\xi, 0}\right|_{\infty} \\
& =C \sum_{x_{1} \cdots 0}^{p}\left|\partial^{\alpha_{1}} / \partial x_{1}^{\alpha_{1}} \exp \left[-i \xi_{x_{1}}\right]\right|_{\infty}=C P(|\xi|),
\end{aligned}
$$

where $P(t) \equiv z_{z_{1}} n t^{x_{i}}$. Similarly $\|F(0, \eta)\| \leqq C P(|\eta|)$. Thus $\|F(\xi, \eta)\| \leqq$ $C^{2} P^{2}(|\xi+i \eta|)$, from which (e) follows. 
REMARK. As a corollary of (e), we can replace the space $\mathscr{D}\left(R^{2}\right)$ by the space $\mathscr{S}\left(R^{2}\right)$ in part (d).

The next theorem shows that the analysis can be carried to complex values of $\eta$ if the support of $T$ is real, or equivalently, if $T$ represents an operator with real spectrum (cf. [6, Theorem 4]).

THEOREM 3. Let $T$ be a spectral distribution in $X$ which has real support. Then

(a) For $(\xi, \eta) \in R \times C$ (i.e., $\xi$ real, $\eta$ complex) there is a function $f_{\xi, \eta}(\cdot) \in \mathscr{B}\left(R^{2}\right)$ satisfying equation (i) near supp $T$.

(b) The function $F(\xi, \eta) \equiv T_{f_{\xi, \eta}}$ is independent of the choice of $f_{\xi, \eta}$ in (a), and is a strongly continuous function of $(\xi, \eta) \in R \times C$.

(c) There is a number $C>0$ and a positive integer $N$ such that

$$
\|F(\xi, \eta)\| \leqq C(1+|\xi+i \operatorname{Re} \eta|)^{N}(1+|\operatorname{Im} \eta|)^{N}
$$

for all $(\xi, \eta) \in R \times C$.

PROof. (a) Let $\varphi \in \mathscr{D}\left(R^{1}\right)$ be a test function which is identically 1 near the origin in $R^{1}$. Define $f_{\xi, \eta}\left(x_{1}, x_{2}\right)=\exp \left[-i\left(x_{1} \xi+\lambda_{2} \eta\right)\right] \cdot \varphi\left(x_{2}\right)$ for all $\left(x_{1}, x_{2}\right) \in R^{2}$.

(b) The strong continuity is proved analogously to Theorem 2(b).

(c) With $\varphi$ as above, define a mapping $u: \mathscr{E}^{\mathscr{C}}\left(R^{1}\right) \rightarrow \mathscr{L}^{\prime}(X)$ as follows. For $\chi$ in the space $\mathscr{E}\left(R^{1}\right)$, let $\chi_{\varphi}\left(x_{1}, x_{2}\right) \equiv \chi\left(x_{2}\right) \cdot \varphi\left(x_{2}\right)$. Then $\chi_{\varphi} \in \mathscr{B}\left(R^{2}\right)$, so that we can define $u(\chi) \equiv T_{\chi_{\varphi}}$. The correspondence $\chi \rightarrow u(\chi)$ is clearly continuous with respect to the usual topology of $\mathscr{E}^{\prime}\left(R^{1}\right)$. Furthermore, if $0 \notin \operatorname{supp} \chi$, then the real axis does not intersect supp $\chi_{i p}$, so $u(\chi)=T_{\chi_{\varphi}}=0$. Thus supp $u \subset\{0\}$. Setting $t \equiv \operatorname{Im} \eta$, define $\chi_{0}\left(x_{2}\right) \equiv\left(t x_{2}\right)^{4+1}$, where $q$ is the order of $u$. Then $\chi_{0}$ vanishes, with all derivatives of order $\leqq q$, on $\{0\} \supset$ supp $u$. By the operator-valued analogy of [2, Theorem 1.5.4] $T_{\left(x_{0}\right) \varphi}=$ $u\left(\chi_{0}\right)=0$; i.e., $T_{\left(t x_{2}\right)^{q+1} \varphi}=0$. Since $\left(t x_{2}\right)^{q+1} \varphi=\left(t x_{2} q\right)^{q+1}$ near the real axis $\left(x_{2}=0\right)$, we have $\left(T_{t x_{2} \varphi}\right)^{q+1}=T_{\left(t x_{2} \varphi\right)^{q+1}}=0$, so that

$$
\exp \left(T_{t x_{2} \varphi}\right)=\sum_{n=0}^{q} \frac{\left(T_{t x_{2} \varphi}\right)^{n}}{n !}
$$

On the other hand, the series for $\exp \left(t x_{2} \varphi\right)$ converges in $\mathscr{B}\left(R^{2}\right)$, so $\exp \left(T_{t x_{2} \varphi}\right)=T_{\exp \left(t x_{2} \varphi\right)}$. But $T_{\operatorname{cxp}\left(t x_{2} \varphi\right)}=F(0, i t)$, so

$$
\begin{aligned}
\|F(0, i t)\| & =\left\|\exp \left(T_{t x_{2} \varphi}\right)\right\|=\left\|\sum_{n=0}^{n} \frac{\left(T_{t x_{2} \varphi}\right)^{n}}{n !}\right\| \\
& \leqq \sum_{n=0}^{q} \frac{|t|^{n}}{n !}\left\|T_{x_{2} \varphi}\right\|^{n} \leqq\left((1+|t|)^{\prime}\right.
\end{aligned}
$$

for all $t \in R^{1}$ and for some $C>0$. 
Now $F(\xi, \eta)=F(\xi, \operatorname{Re} \eta) F(0, i t)$, so the desired estimate follows from (iii) and Theorem 2(e).

\section{The domain of the associated generalized scalar.}

TheOREM 4. Le't A be a generalized scalar operator in $X$ [6, Definition 3] with spectral distribution $T$. Then for a vector $x \in X$, $x$ is in the domain $D_{A}$ of $A$ if and only if $(\partial / \partial \xi) F(\xi, \eta) x$ and $(\partial / \partial \eta) F(\xi, \eta) x$ exist for all $(\xi, \eta) \in$ $R^{2}$. For $x \in D_{A}$, we have

$$
A x=\left.i(\partial / \partial \xi) F(\xi, \eta) x\right|_{\xi=\eta=0}-\left.(\partial / \partial \eta) F(\xi, \eta) x\right|_{\xi=\eta=0} .
$$

To prove this theorem we need the following two lemmas.

Lemma A. For all $x \in D_{A}$, limit ${ }_{n} T_{x_{1} \varphi_{n}} x$ and $\operatorname{limit}_{n} T_{x_{2} \varphi_{n}} x$ exist, where $\left\{\varphi_{n}\right\}$ are as usual.

Lemma B. Le't $U, V: R^{2} \rightarrow X$ be continuous mappings into the Banach space $X$. Assume

$$
-\int l \cdot\left(\partial / \partial x_{1}\right) \varphi d x_{1} d x_{2}=\int \varphi \cdot V d x_{1} d x_{2}
$$

for all $\varphi \in \mathscr{L}\left(R^{2}\right)$. Then $\left(\partial / \partial x_{1}\right) U$ exists for all $\left(x_{1}, x_{2}\right) \in R^{2}$, and equals $V$.

Proof of Lemma A. The double sequence of functions $\psi_{n m} \equiv$ $\left(x_{1} /\left(x_{1}+i x_{2}\right)\right)\left(\varphi_{n}-q_{m}\right)$ is $\mathscr{B}\left(R^{2}\right)$-bounded, and $\inf \left\{|\lambda|: \lambda \in \operatorname{supp} \psi_{n m}\right\} \rightarrow_{n m}$ $\infty$. Thus $\left\|T_{x_{1} \varphi_{n}} x-T_{x_{1} \varphi_{m}} x\right\|=\left\|T_{\lambda \varphi_{n m}} x\right\| \rightarrow_{n m} 0$. Similarly for the other limit. For obvious reasons, we define

$$
(\operatorname{Re} A) x \equiv \underset{n}{\operatorname{limit}} T_{x_{1} \varphi_{n}} x,(\operatorname{Im} A) x \equiv \underset{n}{\operatorname{limit}} T_{x_{2} \varphi_{n}} x .
$$

These definitions are independent of the choice of $\left\{\varphi_{n}\right\}$.

The proof of Lemma $B$ is analogous to [2, Theorem 1.4.2].

Proof of TheOrim 4. Assume $x \in D_{A}$. Then for any $\psi \in \mathscr{D}\left(R^{2}\right)$, we have

$$
-\int \frac{\partial}{\partial x_{1}} \varphi\left(x_{1}, x_{2}\right) F\left(x_{1}, x_{2}\right) x d x_{1} d x_{2}=\hat{T}_{-\partial \varphi / \partial x_{1}} x=T_{-(\partial \varphi)^{\wedge} / \partial x_{1}} x=T_{-i \xi \hat{\varphi}} x
$$

(where $[\xi \hat{\varphi}](\xi, \eta) \equiv \xi \hat{q}(\xi, \eta)$ for all $\left.(\xi, \eta) \in R^{2}\right)$

$$
\begin{aligned}
& =\underset{n}{\operatorname{limit}} T_{\varphi_{n}} T_{\cdots \hat{\xi} \hat{\varphi}} x=\underset{n}{\operatorname{limit}} T_{-i \xi \varphi_{n \hat{\varphi}}} x=-i \operatorname{limit}_{n} T_{\hat{\varphi}} T_{: \varphi_{i:}} x \\
& =-i T_{\hat{\varphi}}(\operatorname{Re} A) \cdot x=-i \int \phi\left(x_{1}, x_{2}\right) F\left(x_{1}, x_{2}\right)(\operatorname{Re} A) x d x_{1} d x_{2} .
\end{aligned}
$$

Lemma B implies

(v) $\quad(\partial / \partial \xi) F\left(\xi, \mu=-i F(\xi, \eta)(\operatorname{Re} A) x\right.$ for all $(\xi, \eta) \in R^{2}$ 
Similarly,

$$
(\partial / \partial \eta) F(\xi, \eta) x=-i F(\xi, \eta)(\operatorname{Im} A) x \quad \text { for all }(\xi, \eta) \in R^{2}
$$

Results (v) and (vi) in turn imply

$$
\begin{aligned}
A x & =(\operatorname{Re} A) x+i(\operatorname{Im} A) x=F(0,0)(\operatorname{Re} A) x+i F(0,0)(\operatorname{Im} A) x \\
& =\left.i(\partial / \partial \xi) F(\xi, \eta) x\right|_{\xi=\eta=0}-\left.(\partial / \partial \eta) F(\xi, \eta) x\right|_{\xi=\eta=0} .
\end{aligned}
$$

We will show that the existence of

$$
\left.(\partial / \partial \xi) F(\xi, \eta) x\right|_{\xi=\eta=0} \text { and }\left.(\partial / \partial \eta) F(\xi, \eta) x\right|_{\xi=\eta=0}
$$

implies $x \in D_{A}$. Let $y=\left.(\partial / \partial \xi) F(\xi, \eta) x\right|_{\xi=\eta=0}$. Then $(\partial / \partial \xi) F(\xi, \eta) x=$ $F(\xi, \eta) y$, and for $\varphi \in \mathscr{D}\left(R^{2}\right)$ we have

$$
\begin{aligned}
T_{\varphi} y & =\hat{T}_{\psi} y \quad\left(\text { where } \psi \in \mathscr{S}\left(R^{2}\right), \hat{\psi}=\varphi\right) \\
& =\int \psi(\xi, \eta) F(\xi, \eta) y d \xi d \eta=\int \psi(\xi, \eta)(\partial / \partial \xi) F(\xi, \eta) x d \xi d \eta \\
& =-\int(\partial / \partial \xi) \psi(\xi, \eta) F(\xi, \eta) x d \xi d \eta=\hat{T}_{-\partial \psi / \partial \xi} x \\
& =T_{-(\partial \psi) \wedge / \partial \xi} x=T_{-i x_{1} \hat{\psi}} x=-i T_{x_{1} \varphi} x
\end{aligned}
$$

Similarly, if $z=\left.(\partial / \partial \eta) F(\xi, \eta) x\right|_{\xi=\eta=0}$ then $T_{\varphi} z=-i T_{x_{2} \varphi} x$. Thus $T_{\lambda \varphi} x=$ $i T_{\varphi} y-T_{\varphi} z$. Now the vanishing of $T$ to power 0 at infinity implies $T$ vanishes to power 1 at infinity for $x$ : i.e., $x \in D_{A}$.

REMARK. If supp $T$ is real, then the function $g_{\xi}(t) \equiv F(\xi, i t)$ is differentiable with respect to $t \in R$, for any $\xi \in R$, in the uniform operator topology. This follows from the representation $F(0, i t)=T_{\exp \left(t x_{2} \varphi\right)}$ for $\varphi$ a test function identically 1 near the origin in $R^{\mathbf{1}}$. (Cf. proof of Theorem 3(c).)

Generalized pseudo-hermitian operators.

Definition. A mapping $A$ of a Banach space $X$ into itself is called generalized pseudo-hermitian (g.p.h.) if

(a) $A$ is a (not necessarily bounded) generalized scalar [6, Definition 3], and

(b) The spectrum $\sigma(A)$ of $A$ is real.

REMARK. [6, Theorem 4] implies that supp $T$ is real for any spectral distribution $T$ which is admitted by a g.p.h. operator $A$.

Definition. A group of operators $\left\{U_{t}\right\}_{t \in R}$ in a Banach space $X$ is called temperate if there are a number $C>0$ and a positive integer $N$ such that $\left\|U_{t}\right\| \leqq C(1+|t|)^{N}$ for all $t \in R$. 
TheOREm 5. Let A be a g.p.h.operator in $X$. Then $-i \cdot A$ is the infinitesimal generator of a temperate $C_{0}$ group of operators in $X$.

Proof. Let $T$ be a spectral distribution which $A$ admits. Then supp $T$ is real. Let $F(\xi, \eta)$ be as in Theorem 3. Define $U_{t} \equiv F(t, i t)$. By Theorem 2(c), $U_{0}=I$. By Theorem $3(\mathrm{~b}), U_{t}$ is strongly continuous. The group property $U_{t+s}=U_{t} U_{s}$ is clear. By Theorem $3(\mathrm{c}),\left\|U_{t}\right\| \leqq C(1+|t|)^{2 N}$, so $U_{t}$ is a temperate $C_{0}$ group. Let $B$ denote the infinitesimal generator of $U_{t}$. If $x \in D_{A}$, then Theorem 4 (together with the following remark) implies the existence of $(d / d t)\left(U_{t} x\right)$ :

$$
\begin{aligned}
\left.(d / d t)\left(U_{t} x\right)\right|_{t=0} & =\left.(\partial / \partial \xi) F(\xi, \eta) x\right|_{\xi=\eta=0}+\left.(d / d t)[F(0, i t) x]\right|_{t=0} \\
& =-i(\operatorname{Re} A) x+\left.(d / d t)\left[T_{\exp \left(t x_{2} \varphi\right)} x\right]\right|_{t=0} \\
& =-i(\operatorname{Re} A) x+T_{x_{2} \varphi} x \\
& =-i(\operatorname{Re} A) x+(\operatorname{Im} A) x=-i \cdot A x .
\end{aligned}
$$

Thus $x \in D_{B}$ and $B x=-i \cdot A x$.

On the other hand, let $x \in D_{B}$. Then the existence of the derivative $\left.(\partial / \partial \xi) F(\xi, \eta) x\right|_{\xi=\eta=0}$ follows from the relation

$$
F(\xi, 0) x=F(0,-i \xi) F(\xi, i \xi) x,
$$

remembering the remark after Theorem 4 and the differentiability of $F(\xi, i \xi) x=U_{\xi} x$ with respect to $\xi$. The derivative $(\partial / \partial \eta) F(0, \eta)$ exists in the uniform operator topology since supp $T$ real implies that $F(0, \eta)=T_{\exp \left(-i x_{2} \eta \varphi\right)}$. Thus Theorem 4 implies $x \in D_{A}$, and we have $B=-i \cdot A$.

\section{REFERENCES}

1. N. Dunford and J. T. Schwartz, Linear operators. I: General theory, 2nd ed., Interscience, New York, 1964.

2. L. Hörmander, Linear partial differential operators, Die Grundlehren der math, Wissenschaften, Band 116, Academic Press, New York; Springer-Verlag, Berlin, 1963. MR 28 \#4221.

3. J. Horváth, Topological vector spaces and distributions. Vol. I, Addison-Wesley, Reading, Mass., 1966. MR 34 \#4863.

4. B. Kritt, Spectral decomposition of positive and positive-definite distributions of operators, Bull. Acad. Polon. Sci. Sér. Sci. Math. Astronom. Phys. 16 (1968), 865-870. MR 39 \#3307.

5. - Generalizea' pseudo-Hermitian operators, Proc. Amer. Math. Soc. 30 (1971), 343-348.

6. - A theory of unbounded generalized scalar operators, Proc. Amer. Math. Soc. 32 (1972), 484-490.

Department of Mathematics, University of Baltimore, Baltimore, Maryland 21201 